\title{
Potential Role of the Galectin-9/TIM-3 Axis in the Disparate Progression of SARS-CoV-2 in a Married Couple: A Case Report
}

\author{
Alejandro Martín-Quirós ${ }^{\mathrm{a}} \quad$ Charbel Maroun-Eid $^{\mathrm{b}} \quad$ José Avendaño-Ortiz ${ }^{\mathrm{b}, \mathrm{c}}$ \\ Roberto Lozano-Rodríguez ${ }^{b, c}$ Jaime Valentín Quirogab,c Verónica Terrón ${ }^{b, c}$ \\ Karla Montalbán-Hernández ${ }^{\text {b, c }}$ Miguel A. García-Garrido ${ }^{a}$ Elena Muñoz del Vala \\ Álvaro del Balzo-Castillo ${ }^{\text {b, c }}$ Carolina Rubio ${ }^{\text {b, c }}$ Carolina Cubillos-Zapata ${ }^{d}$ Luis A. Aguirre $^{\text {b, c }}$ \\ Eduardo López-Collazo b, c, d \\ aEmergency Department and Emergent Pathology Research Group, IdiPAZ La Paz University Hospital, Madrid, Spain; \\ b The Innate Immune Response Group, IdiPAZ, La Paz University Hospital, Madrid, Spain; 'Tumor Immunology Laboratory, \\ IdiPAZ, La Paz University Hospital, Madrid, Spain; ${ }^{d}$ CIBER of Respiratory Diseases (CIBERES), Madrid, Spain
}

\section{Established Facts}

- It is already known that different previous health statuses could affect illness progression in COVID-19 patients.

- It is also known that a cytokine storm takes place in patients with COVID-19 who exhibit a poor prognosis.

\section{Novel Insights}

- The Gal-9/TIM-3 axis could be crucial for the stratification of COVID-19 patients with a poor prognosis.

\section{Keywords}

COVID-19 - Immune checkpoints · Galectin-9 · TIM-3 ·

SARS-CoV-2 $\cdot$ Sepsis

\section{Abstract}

We report the disparate clinical progression of a couple infected by SARS-CoV-2 based on their immune checkpoint (IC) levels and immune cell distribution in blood from admis- sion to exitus in patient 1 and from admission to discharge and recovery in patient 2 . A detailed clinical follow-up accompanied by a longitudinal analysis of immune phenotypes and IC levels is shown. The continuous increase in the soluble IC ligand galectin-9 (Gal-9) and the increment in Tcell immunoglobulin and mucin domain-containing 3 (TIM-

A.M.-Q. and C.M.-E. contributed equally to this work. karger@karger.com www.karger.com/bmh

Karger $\stackrel{\text { ' }}{5}$

BOPEN ACCESS
(C) 2021 The Author(s)

Published by S. Karger AG, Basel

This is an Open Access article licensed under the Creative Commons Attribution-NonCommercial-4.0 International License (CC BY-NC) (http://www.karger.com/Services/OpenAccessLicense), applicable to the online version of the article only. Usage and distribution for commercial purposes requires written permission.
Correspondence to:

Eduardo López-Collazo, elopezc@ salud.madrid.org 
3) protein in T cells in patient 1 suggests an activation of the Gal-9/TIM-3 axis and, subsequently, a potential cell exhaustion in this patient that did not occur in patient 2. Our data indicate that the Gal-9/TIM-3 axis could be a potential target in this clinical setting, along with a patent effector memory T-cell reduction.

(c) 2021 The Author(s)

Published by S. Karger AG, Basel

\section{Introduction}

The first clinical data obtained from patients with COVID-19 suggested that SARS-CoV-2 has a dramatic impact on the immune system $[1,2]$. According to several reports, severe effects in patients with COVID-19 appear about 8-11 days after disease onset, when proinflammatory cytokines reach their peaks of expression [3]. In line, the total number of lymphocytes is significantly reduced in those patients with a poor prognosis $[1,4]$. These data suggest an impairment of the immune system, allowing secondary infections $[5,6]$. T-cell exhaustion and increased inhibitory receptor expression on peripheral $\mathrm{T}$ cells have been reported in this disease [7-9]. Moreover, although there is evidence of T-cell activation in COVID-19 patients [10], several studies have found polyfunctionality or cytotoxicity decreases [6, 11]. Like during sepsis, the potential role of immune checkpoints (ICs) and their ligands in the lymphocytopenia observed in severe COVID-19 patients have been partially studied $[5,10,12]$. Besides, how this activation should be viewed in the context of COVID-19 lymphopenia remains unclear. Here, we present a married couple with COVID-19 who exhibited remarkably disparate clinical progression. Both patients had the same SARSCoV-2 exposure, and the same clinical presentation at onset, with bilateral interstitial pneumonia, respiratory failure, and similar laboratory test results. However, their clinical evolution and outcome were completely different. A longitudinal analysis of their IC levels and immune cell distribution in blood showed a differential profile that could provide an explanation for the unequal evolution in these patients.

Fig. 1. Timelines of progression of the clinical courses of the 2 patients with confirmed SARS-CoV-2 infection. Red boxes show the events for patient 1 and green boxes show the events for patient 2 . $\mathrm{ABL}$, amphotericin B liposomal; ARDS, acute respiratory distress syndrome; CT, computed tomography; ED, emergency department; $\mathrm{FiO}_{2}$, fraction of inspired oxygen; HCQ, hydroxychloro-

\section{Case Description, Diagnostic Assessment, Therapeutic Intervention, Follow-Up, and Outcomes}

A Caucasian married couple in Madrid (Spain) were both diagnosed with COVID-19 through a SARS-CoV-2 RT-PCR test. Four days before the onset of symptoms, the couple had had close contact with the husband's mother, who was diagnosed with COVID-19 and died (see evolution in Fig. 1).

Patient 1 was a 60-year-old, retired, overweight man with hypertension, type 2 diabetes, type 1a hemochromatosis with no hepatic or systemic involvement, and a history of penicillin allergy. He presented no records of previous surgical interventions. Symptom onset was on April 22, 2020, with mild influenza-like illness (wet cough, fever, asthenia, and hyporexia). He was referred to the emergency department (ED) of a secondary hospital, where he was diagnosed with possible COVID-19. His blood tests and chest Xray were compatible with mild disease, and he was discharged with a prescription for $400 \mathrm{mg}$ cefditoren twice a day (b.i.d.), $500 \mathrm{mg}$ azithromycin 4 times a day (q.i.d.), $400 \mathrm{mg}$ hydroxychloroquine b.i.d. the first day and $200 \mathrm{mg}$ b.i.d. for 4 more days, and $60 \mathrm{mg}$ prednisone q.i.d. for 3 days.

Due to impairment from dyspnea, the patient was admitted to our ED on May 1, 2020. The physical examination showed mild tachypnea, tachycardia, and disseminated crackling rales on pulmonary auscultation. Table 1 shows the baseline variables and vital signs. He was diagnosed with respiratory failure, with an oxygen saturation of $89 \%$ and bilateral interstitial pneumonia (online suppl. Fig. S1; for all online suppl. material, see www. karger.com/doi/10.1159/000514727). An RT-PCR for SARSCoV-2 confirmed the COVID-19 etiology. We added tocilizum$\mathrm{ab}$ and low-molecular-weight heparin (LMWH) at prophylactic doses to his previous therapy with hydroxychloroquine and prednisone. The patient was admitted to the internal medicine ward. His respiratory status worsened despite methylprednisolone boluses ( $250 \mathrm{mg}$ q.i.d. for 3 days) and lying in the prone position, and he was finally admitted to the intensive care unit (ICU) on May 9 with COVID-19-related acute respiratory distress syndrome. Empirical treatment with aztreonam and linezolid was administered until May 20. A point-of-care echocardiogram suggested a pulmonary embolism, and the patient was therefore administered fibrinolysis, resulting in mild improvement of the respiratory status. LMWH was adjusted to a therapeutic dosage, and the patient was placed on mechanical ventilation on May 10. Although the patient was administered therapeutic doses of LMWH, he developed an acute distal left leg arterial occlusion, which was treated conservatively. During his ICU stay, the patient required noradrenaline at varying dosages. The results of his systematic microbiological workup were repeatedly negative, except on May 21 (when Enterococcus faecalis was found in the urine culture during a febrile episode) and May

quine; LMWH, low-molecular-weight heparin; Lop/R, lopinavir/ ritonavir; MPS, methylprednisolone; MSSA, methicillin-sensitive Staphylococcus aureus; MV, mechanical ventilation; OS, oxygen saturation; PE, pulmonary embolism; q.i.d., 4 times a day; QTc, corrected QT interval; TC, tocilizumab.

(For figure see next page.) 


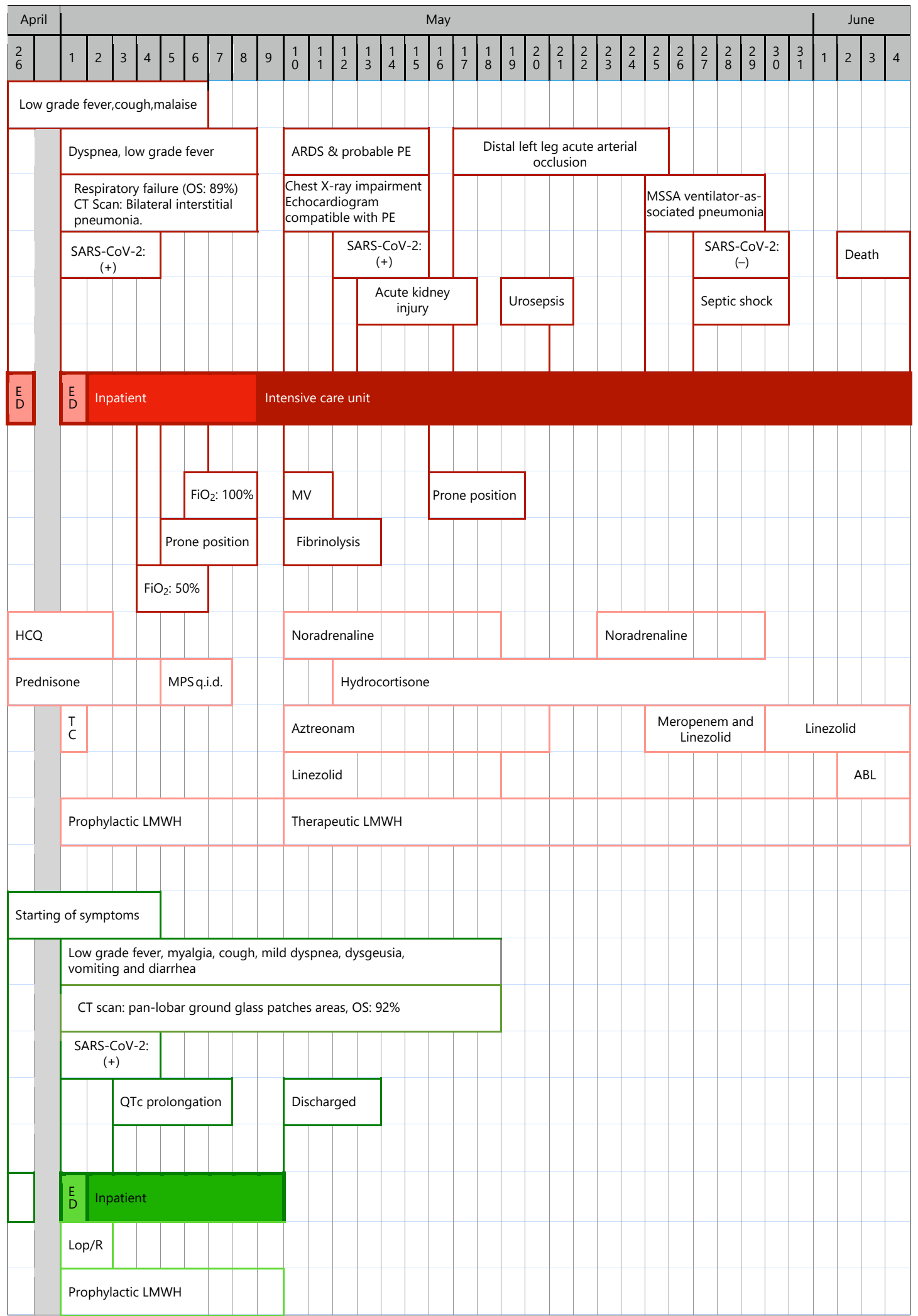


25 (when methicillin-sensitive Staphylococcus aureus was isolated in the bronchoalveolar lavage and blood cultures taken due to his impaired status). Sepsis with ventilator-associated pneumonia and secondary bacteremia were diagnosed. The patient was administered meropenem and linezolid empirically from May 25 and maintained after microbiological results showed that both microorganisms were sensitive. The patient developed refractory septic shock and was treated with hydrocortisone, high-dose noradrenaline, and empirical liposomal amphotericin B. On May
25, the SARS-CoV-2 RT-PCR was negative. The patient eventually died on June 4 after 40 days of illness.

Patient 2 was a 60 -year-old, non-obese woman without previous conditions or surgeries. On April 22, 2020, she started with mild symptoms characterized by asthenia, myalgia, low-grade fever, wet cough, moderate dyspnea, dysgeusia, nausea with various episodes of vomiting, and diffuse abdominal discomfort with diarrhea. In the ED, she presented tachycardia (111 bpm) and mild tachypnea, with an oxygen saturation of $92 \%$. Table 1 shows her

Table 1. Patients' and HVs' baseline clinical characteristics

\begin{tabular}{llll}
\hline & Patient 1 & Patient 2 & HVs $(n=10)$ \\
\hline Age, years & 60 & 60 & $56.1(9.0)$ \\
Gender & Male & Female & 5 female, 5 male \\
Weight, kg & 80 & 56 & $76.1(5.2)$ \\
Height, cm & 178 & 161 & $174.4(23.2)$ \\
BMI, kg/m ${ }^{2}$ & 25.2 & 21.6 & $24.8(4.4)$ \\
Comorbidities & & & \\
$\quad$ Type 2 diabetes & Yes & No & No \\
$\quad$ Hypertension & Yes & No & No \\
$\quad$ Other & No & No & No \\
Temperature, ${ }^{\circ}$ C & 36.9 & 36.3 & $36.1(0.4)$ \\
O saturation, $\%$ & 89 & 91 & $96.8(3.1)$ \\
Ferritin, ng/mL & 550 & 653 & $215(42.1)$ \\
Lactate, nmol/L & 3.1 & 2 & $0.8(0.3)$ \\
LDH, IU/L & 289 & 350 & $258(37)$ \\
GOT, IU/L & 20 & 55 & $32(9.7)$ \\
GPT, IU/L & 25 & 75 & $36(7.4)$ \\
pH & 7.41 & 7.44 & $7.41(0.2)$ \\
CRP, mg/L & 9.8 & 7.9 & $5.6(3.8)$ \\
MBP, mm Hg & 75 & 75 & $73(12)$ \\
SBP, mm Hg & 125 & 123 & $125(10)$ \\
Heart rate, bpm & 105 & 111 & $87(14)$ \\
Respiratory rate, brpm & 28 & 28 & $15(4)$ \\
Pneumonia at admission & Yes & Yes & No \\
qSOFA score & 1 & 1 & - \\
CGS score & 15 & 15 & - \\
SARS-CoV-2 (RT-PCR) & Positive & Positive & Negative \\
\hline
\end{tabular}

Values for HVs denote mean (SD). bpm, beats per minute; BMI, body mass index; brpm, breaths per minute; GCS, Glasgow Coma Score; CRP, C-reactive protein; GOT, glutamic oxaloacetic transaminase; GPT, glutamicpyruvic transaminase; $\mathrm{HV}$, healthy volunteer; $\mathrm{LDH}$, lactate dehydrogenase; $\mathrm{MBP}$, mean blood pressure; qSOFA, quick Sequential Organ Failure Assessment; SBP, systolic blood pressure; SD, standard deviation.

Fig. 2. Gal-9/TIM-3 axis showing disparate levels and patterns for patients 1 and 2. a The plasma levels of soluble Gal-9 (sGal-9) from patients 1 and 2, collected until exitus or discharge, respectively, and the mean levels for 10 healthy volunteers (HVs) with negative SARS-CoV-2 RT-PCR results are shown. b A final reading from patient 2, obtained 70 days after disease onset, is also shown. The mean fluorescence intensities (MFI) of TIM- 3 on gated $\mathrm{CD}^{+}$cells are shown. $\mathbf{c}$ Percentage of TIM $-3^{+}$cells on gated $\mathrm{CD} 3^{+}$cells. $\mathbf{d}$ The percentages of $\mathrm{CD}^{+}$cells from total leukocytes are shown. e, $\mathbf{f}$ The percentages of $\mathrm{CD}^{+}(\mathbf{e})$ and $\mathrm{CD}^{+}(\mathbf{f})$ cells on $\mathrm{CD}^{+}$cells are shown. $\mathbf{g}, \mathbf{h}$ The MFI of TIM-3 (g) and the percentage of TIM- $3^{+}$ cells $(\mathbf{h})$ on gated CD $4^{+}$cells are shown. $\mathbf{i}, \mathbf{j}$ The MFI of TIM-3 (i) and the percentage of TIM- $3^{+}$cells $(\mathbf{j})$ on gated $\mathrm{CD} 8^{+}$cells are shown. $\mathbf{k}$ The $t$-SNE map clustering expression of TIM-3 on CD $4^{+}$ and $\mathrm{CD}^{+}$populations is shown. $\mathbf{a}-\mathbf{j}$ Data from patient 1 are shown in red, and data from patient 2 are shown in green. Dashed lines represent the calculated means from the analysis of a single sample from $10 \mathrm{HVs}$ (mean $\pm \mathrm{SD}$ ). Arrows indicate important clinical events (patient 1: ICU admission, sepsis, and septic shock; patient 2: discharge).
(For figure see next page.) 

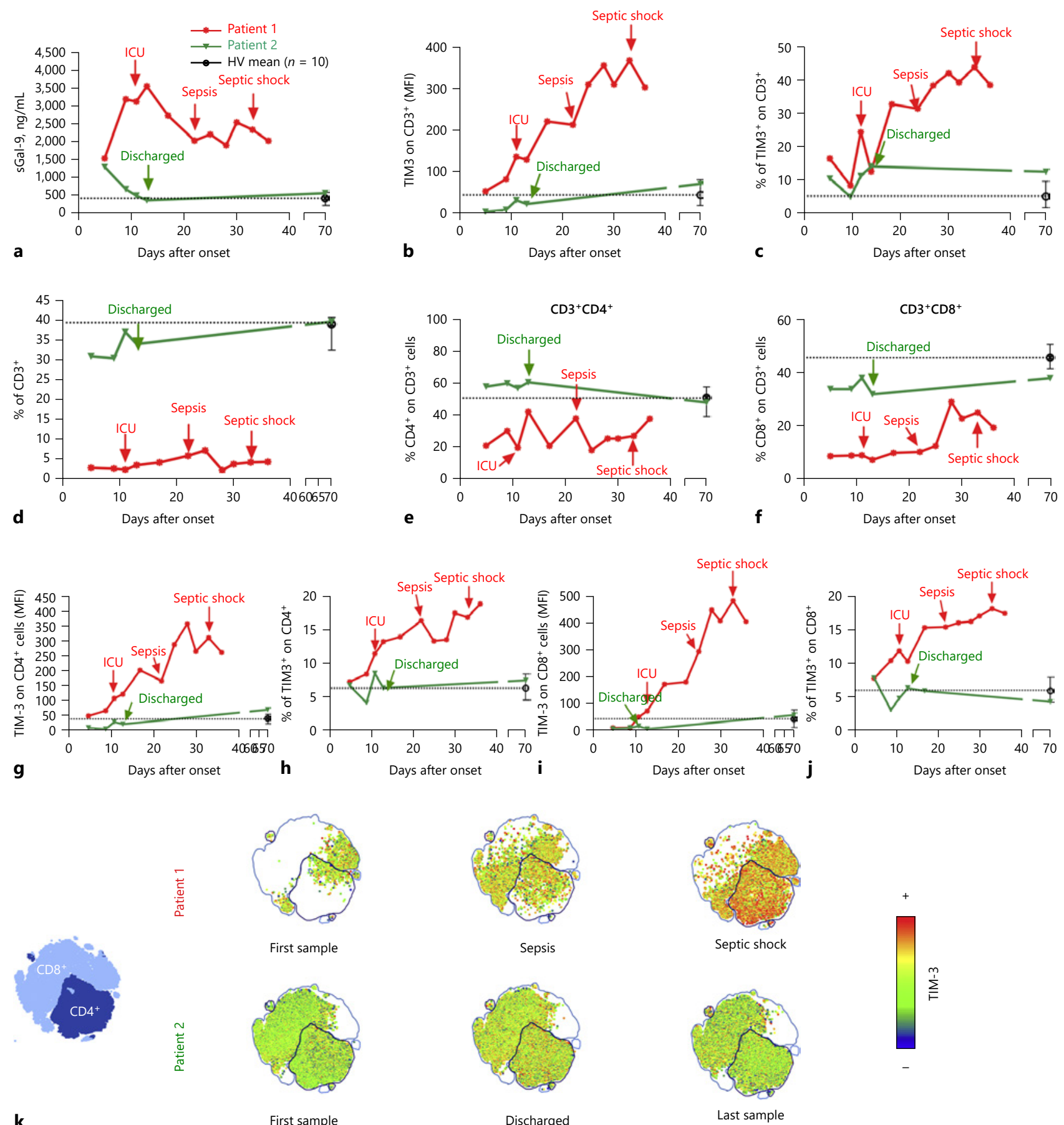

k

First sample

Discharged

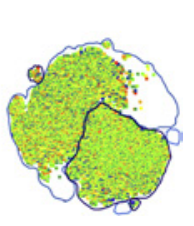



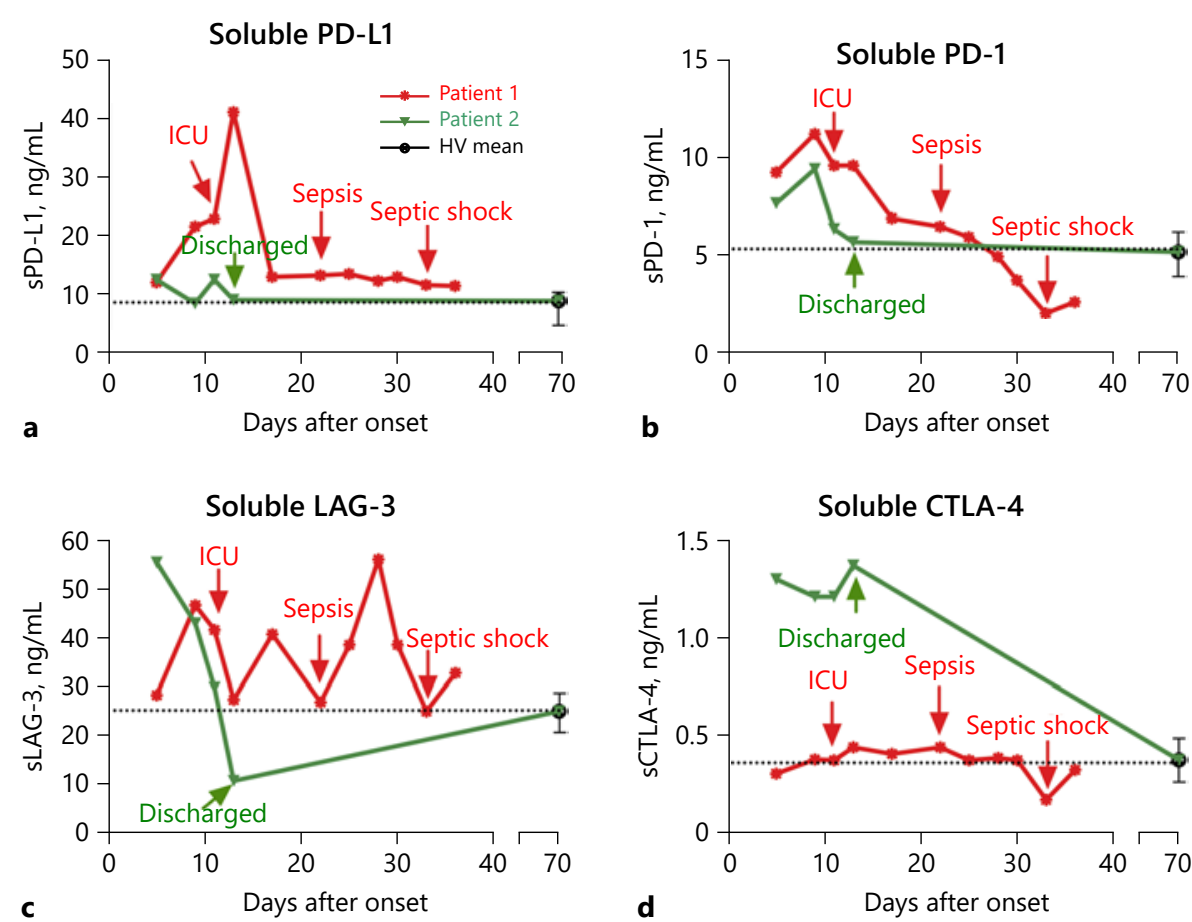

Soluble 4-1BB

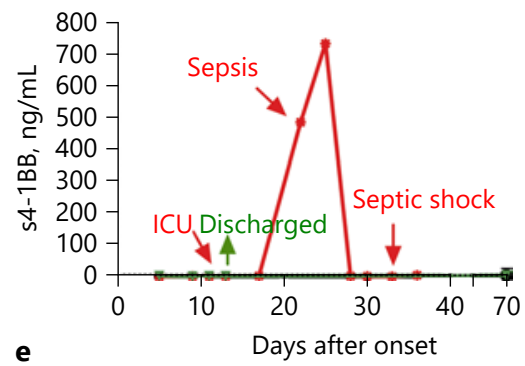

Soluble CD86

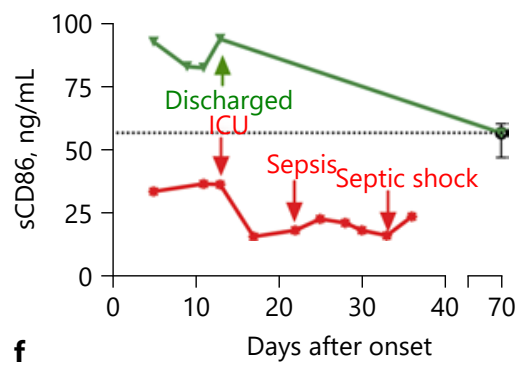

HLA-DR on monocytes

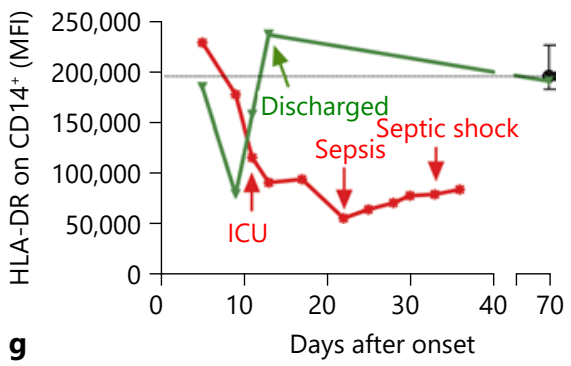

Fig. 3. Soluble plasma levels of immune checkpoints from patients 1 and 2. a-f The plasma levels of soluble immune checkpoints from patients 1 and 2, collected until exitus or discharge, respectively, and the calculated means from the analysis of a single sample from 10 healthy volunteers (HV) with negative SARS-CoV-2 RT-PCR results. A final reading from patient 2, obtained 70 days after disease onset, is also shown. Soluble levels of PD-L1 (a), PD-1 (b), LAG-3 (c), CTLA-4 (d), 4-1BB (e), and CD86 (f) are shown

baseline variables and vital signs. The patient was diagnosed with panlobar COVID-19 pneumonia confirmed by SARS-CoV-2 RTPCR (online suppl. Fig. S2). Blood tests showed a significant increase in her ferritin level, with other parameters within normal limits. The patient was admitted to the internal medicine ward, where she was administered lopinavir/ritonavir (400 mg/100 mg b.i.d.) and a prophylactic dose of LMWH. Lopinavir/ritonavir was discontinued after $48 \mathrm{~h}$ due to QTc interval prolongation. In view of the patient's clinical improvement and having reached 10 days of illness, no other antiviral treatment was started. The patient pro- in the respective panels. $\mathbf{g}$ The mean fluorescence intensities (MFI) of HLA-DR on gated CD14 ${ }^{+}$monocytes are shown. $\mathbf{a}-\mathbf{g}$ Data from patient 1 are shown in red, and data from patient 2 are shown in green. Dashed lines represent the calculated means from the analysis of a single sample from $10 \mathrm{HVs}$ (mean $\pm \mathrm{SD}$ ). Arrows indicate important clinical events (patient 1: ICU admission, sepsis, and septic shock; patient 2: discharge).

gressed adequately and was discharged 9 days after her admission and after 17 days of illness.

\section{Immune Phenotyping and IC Profile}

We repeatedly obtained blood samples from both patients until exitus (patient 1) or discharge (patient 2). In addition, 10 healthy volunteers with a single blood sam- 


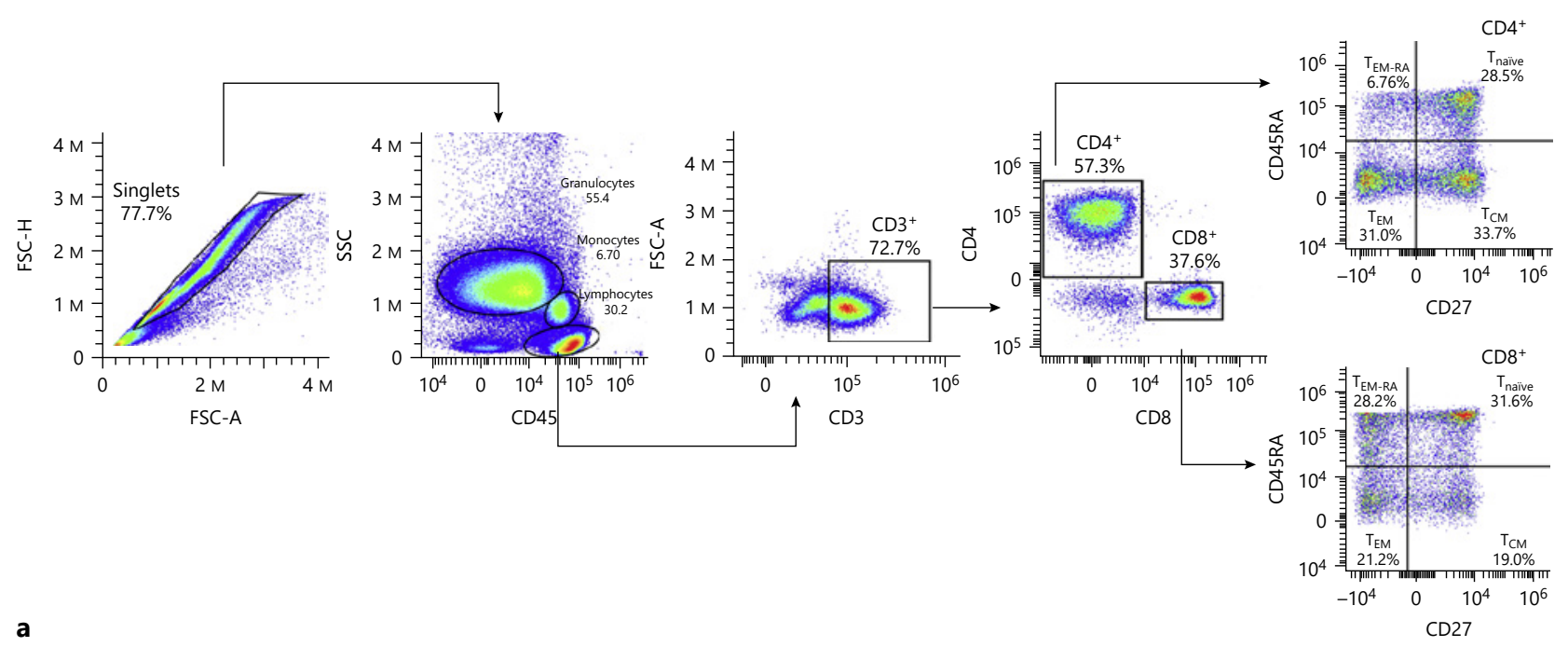

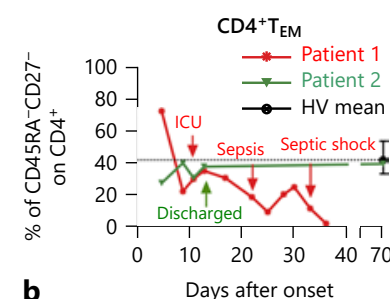

$\mathrm{CD}^{+} \mathrm{T}_{\mathrm{EM}}$

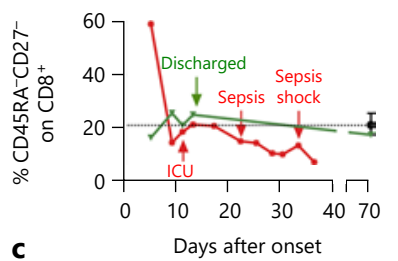

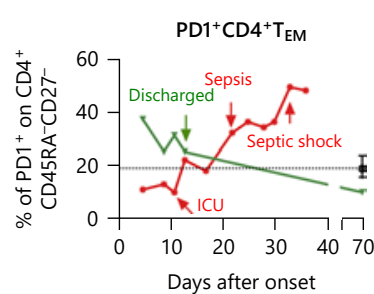

$\mathrm{PD}^{+}{ }^{+} \mathrm{CD} 8^{+} \mathrm{T}_{\mathrm{EM}}$

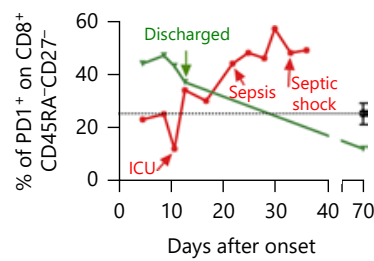

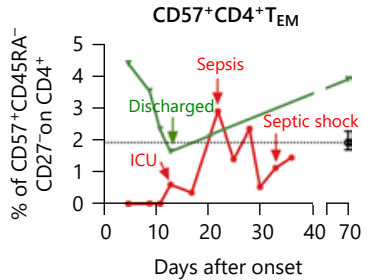

$\mathrm{CD} 57^{+} \mathrm{CD} 8{ }^{+} \mathrm{T}_{\mathrm{EM}}$

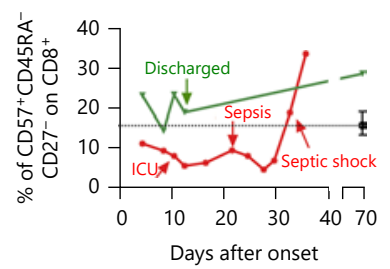

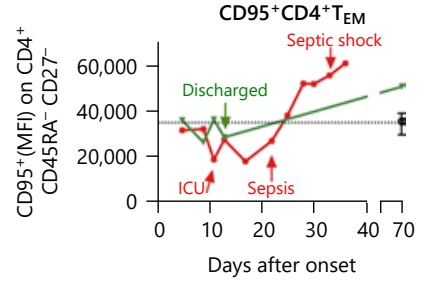

$\mathrm{CD}^{+} 5^{+} \mathrm{CD} 8^{+} \mathrm{T}_{\mathrm{EM}}$

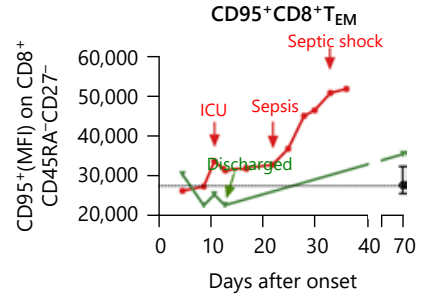

Fig. 4. Effector memory $\mathrm{T}$-cell analysis. a Gating strategy $\left(\mathrm{T}_{\mathrm{EM}}\right.$, effector memory $\mathrm{T}$ cells; $\mathrm{T}_{\mathrm{CM}}$, central memory $\mathrm{T}$ cells; $\mathrm{T}_{\mathrm{EM}-\mathrm{RA}}$, terminally differentiated $\mathrm{T}$ cells; $\mathrm{T}_{\text {naive }}$, naïve $\mathrm{T}$ cells). $\mathbf{b}$, $\mathbf{c}$ The analyses of $\mathrm{T}_{\mathrm{EM}}$ on both gated $\mathrm{CD}^{+}(\mathbf{b})$ and $\mathrm{CD}^{+}(\mathbf{c})$ cells are shown. Left panels: the percentages of $\mathrm{T}_{\mathrm{EM}}$ are shown. Central left panels: the mean fluorescence intensities (MFI) of PD-1 on gated CD4 ${ }^{+}$ and $\mathrm{CD}^{+} \mathrm{T}_{\mathrm{EM}}$ are shown. Central right panels: the percentages of $\mathrm{CD} 7^{+}$cells on gated $\mathrm{CD} 4^{+}$and $\mathrm{CD}^{+} \mathrm{T}_{\mathrm{EM}}$ are shown. Right

ple were included in the study (see their main characteristics in Table 1). From the study of soluble ICs and ligands in plasma, we detected that soluble Gal-9 levels were elevated in both patients at onset. However, a significantly different progression was observed after 8 days of illness (Fig. 2a). This disparity matched the completely different clinical progression. We also studied other soluble ICs and ligands (Fig. 3a-f), reinforcing the findings panels: the MFI of $\mathrm{CD} 95$ on gated $\mathrm{CD} 4^{+}$and $\mathrm{CD} 8^{+} \mathrm{T}_{\mathrm{EM}}$ are shown. b, c Note that data from patient 1 are shown in red, while the data from patient 2 are shown in green. The dashed lines represent the calculated means from the analysis of a single sample from 10 healthy volunteers $(\mathrm{HVs})($ mean $\pm \mathrm{SD})$. Arrows indicate important clinical events (patient 1: ICU admission, sepsis, and septic shock; patient 2: discharge). of disparate immune responses observed in the patients throughout their illness. Interestingly, sCD86 and monocyte expression of HLA (human leukocyte antigen)-DR isotype showed similar patterns, pointing to a drastic reduction in the antigen presentation ability of patient 1 from onset (Fig. 3f, g).

Several previous reports have indicated that interactions between Gal-9 and TIM-3 could result in T-cell ex- 


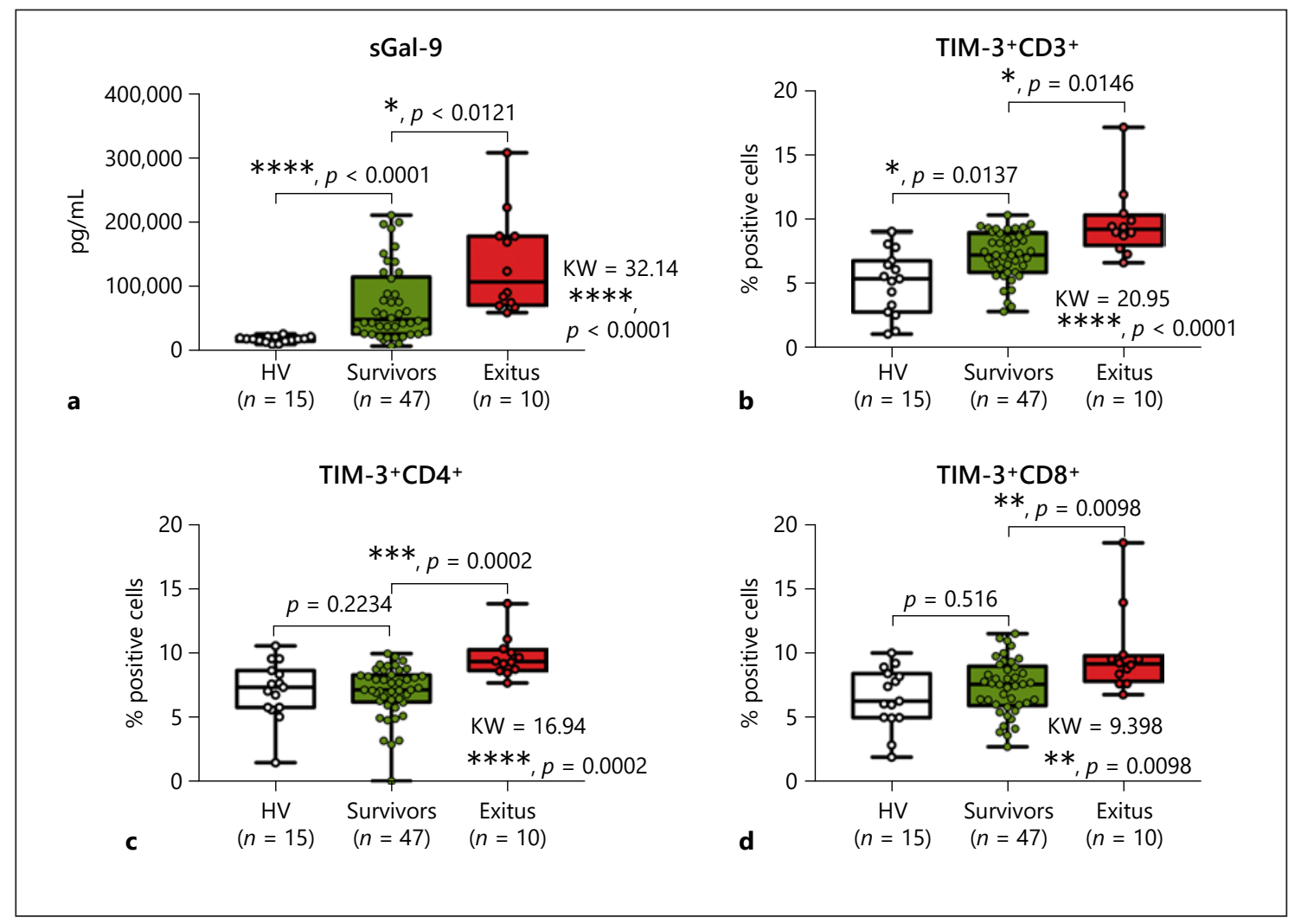

Fig. 5. Gal-9/TIM-3 axis showing disparate levels in patients with different outcomes. Blood samples from 57 COVID-19 patients, recruited on admission and before any treatment, were analyzed. a Plasma levels of soluble Gal-9 (sGal-9) from COVID-19 patients classified according to their outcome (survivors or exitus) and healthy volunteers (HVs) are shown. b-d The percentages of TIM- $3^{+}$cells on $\mathrm{CD}^{+}(\mathbf{b}), \mathrm{CD}^{+}(\mathbf{c})$, and $\mathrm{CD} 8^{+}(\mathbf{d})$ cells are shown. a-d Kruskal-Wallis statistics and multiple comparisons are shown.

haustion and, ultimately, a significant reduction in cell count. Likewise, the progression of TIM-3 expression in $\mathrm{CD}^{+}$cells was remarkably disparate, with an almost exponential increase in patient 1 , while patient 2 maintained the expression of this IC at the levels reported for 10 healthy volunteers (Fig. 2b, c). In addition, the percentages of circulating $\mathrm{CD}^{+}$cells as well as $\mathrm{CD}^{+}$and $\mathrm{CD}^{+}$subpopulations from both patients were different, with a patent low level in patient 1 , which remained low during the whole longitudinal temporal series (Fig. 2d-f). Moreover, TIM-3 levels were markedly higher on both the $\mathrm{CD} 4^{+}$and $\mathrm{CD} 8^{+} \mathrm{T}$ cells from patient 1 compared with patient 2 (Fig. $2 \mathrm{~g}-\mathrm{j}$ ), an effect that was more pronounced over time. $t$-SNE cluster map analysis of TIM-3 expression on $\mathrm{CD}^{+}$and $\mathrm{CD} 8^{+} \mathrm{T}$ cells also illustrated these differences (Fig. 2k).

Due to the impact of effector memory $\mathrm{T}$ cells $\left(\mathrm{T}_{\mathrm{EM}}\right.$, defined as $\mathrm{CD}^{+} \mathrm{CD} 45 \mathrm{RA}^{-} \mathrm{CD} 27^{-}$, see gating strategy in Fig. 4a) on resolving SARS-CoV-2 infection [13], we ana- lyzed their percentages in circulating blood from the study patients. Both $\mathrm{CD} 4^{+}$and $\mathrm{CD} 8^{+} \mathrm{T}_{\mathrm{EM}}$ levels showed a downward curve for patient 1 but not for patient 2 (Fig. 4b, c, left panels). Note that the expression of cell senescence marker $\mathrm{PD}-1$ on $\mathrm{CD} 4^{+} \mathrm{T}_{\mathrm{EM}}$ was added to this effect in the latter points and on $\mathrm{CD}^{+} \mathrm{T}_{\mathrm{EM}}$ some time earlier (Fig. 4b, c, central left panels). The expression of CD57, another reported senescence marker, did not show high levels in patient 1 , except at the final point on $\mathrm{CD}^{+}$ $\mathrm{T}_{\mathrm{EM}}$ (Fig. 4b, c, central right panels). Eventually, upregulation of the proapoptotic factor CD95 was patent on $\mathrm{CD} 8^{+} \mathrm{T}_{\mathrm{EM}}$ from an early point in patient 1 , which was also evident on $\mathrm{CD}^{+} \mathrm{T}_{\mathrm{EM}}$ after sepsis onset (Fig. $4 \mathrm{~b}$, c, right panels). A similar analysis was performed on naïve $\left(\mathrm{CD}^{+}{ }^{+} \mathrm{CD} 45 \mathrm{RA}^{+} \mathrm{CD} 27^{+} ; \mathrm{T}_{\text {naive }}\right)$, central memory $\left(\mathrm{CD}^{+}{ }^{+} \mathrm{CD} 45 \mathrm{RA}^{-} \mathrm{CD} 27^{+} ; \mathrm{T}_{\mathrm{CM}}\right)$, and terminally differentiated $\left(\mathrm{CD}^{+}{ }^{+} \mathrm{CD} 45 \mathrm{RA}^{+} \mathrm{CD} 27^{-}\right.$; $\left.\mathrm{T}_{\mathrm{EM}-\mathrm{RA}}\right) \mathrm{CD}^{+}$and $\mathrm{CD} 8^{+}$ cells (online suppl. Fig. S3). Both $\mathrm{CD} 4^{+}$and $\mathrm{CD} 8^{+} \mathrm{T}_{\text {naïve }}$ percentages increased in patient 1 when he was close to 
the onset of sepsis. In the case of $\mathrm{T}_{\mathrm{CM}}$, an increase in both $\mathrm{CD}^{+}$and $\mathrm{CD}^{+} \mathrm{T}_{\mathrm{CM}}$ levels was detected in patient 1 after the onset of sepsis. Eventually, the analysis of $\mathrm{T}_{\mathrm{EM}-\mathrm{RA}}$ showed a peak increase at the moment close to ICU admission, followed by a pronounced decrease in both $\mathrm{CD} 4^{+}$and $\mathrm{CD} 8^{+}$populations in patient 1.

TIM-3 expression was also evaluated on other immune cells, including $\mathrm{CD}_{14}{ }^{+}$monocytes, natural killer cells, and neutrophils (online suppl. Fig. S4, S5). Note that the interleukin (IL)-6 and IL-17 levels increased in patient 1 approximately 10 days after disease onset (online suppl. Fig. S5G, H).

\section{Levels of Soluble Gal-9 in Plasma and TIM-3 Expression on T Cells from COVID-19 Patients with Disparate Evolution}

To evaluate the potential role of the Gal-9/TIM-3 axis as a biomarker of prognosis in the COVID-19 clinical context, we did a retrospective analysis of samples from COVID-19 patients with disparate evolution. Blood samples taken on admission and before any treatment were stored in the Biobank of La Paz University Hospital. Patients included in the study were classified according to their final outcome as survivors $(n=47)$ or exitus $(n=10)$. Note that new healthy volunteers $(n=15)$ were also included (online suppl. Table 1). As Figure 5 shows, levels of soluble Gal-9 were significantly higher in exitus patients than in survivors. Moreover, expression of TIM-3 on T cells was also higher in those patients with the worst evolution.

\section{Discussion and Conclusion}

We showed the disparate clinical courses of 2 patients with different previous health statuses. Patient 1 had suffered from diabetes and hypertension, which constitute risk factors for complications during COVID-19 infection, mostly for males [14-16]. In contrast, patient 2 had no comorbidities. Infection of both patients took place at the same time and by the same source when community transmission of SARS-CoV-2 was controlled in Madrid (Spain). In this context, any differences in their clinical course cannot be related to different virus strains or doses of infection.

As shown in Table 1, the baseline clinical differences between these two patients lie in the cardiovascular profile. In fact, they could be closely related to our immunological findings, as some evidence regarding the patho- genesis of COVID-19 has suggested [17]. Note that the virus uses angiotensin-converting enzyme 2 as a receptor to enter cells, altering the renin-angiotensin axis [18]. This could lead to an increased angiotensin II effect, including increased inflammation [19]. However, this hypothesis requires further study.

Altogether, the continuous increase in soluble Gal-9 and the increment in TIM-3 on T cells in patient 1 could suggest an activation of the Gal-9/TIM-3 axis and a potential cell exhaustion in this patient, in strict contrast to what happened in patient 2. However, this hypothesis must be confirmed by further mechanistic assays. Additionally, reported data have suggested that $\mathrm{CD}^{+} \mathrm{T}_{\mathrm{EM}}$, one of the most important immune cell populations for resolving infection, would undergo apoptosis during infection. This explains the clinical course of patient 1, including the sepsis and secondary infections suffered by the patient.

According to several authors, TIM-3 and Gal-9 have shown the ability to induce $\mathrm{T}$-cell exhaustion and reduce $\mathrm{CD}^{+}$effector cells [20-22]. In line, some authors have proposed the association of various ICs including soluble TIM3 with COVID-19 severity [9]. Our data suggest that soluble Gal-9 and TIM-3 expression on T cells could be biomarkers for those COVID-19 patients at high risk of suffering from sepsis and secondary infections. In addition, the Gal-9/TIM-3 axis could also play a role in modulating other cell types. For example, TIM-3 expression on neutrophils was shown to be involved in bacterial killing [23]. Along these lines, we found a transient peak of TIM-3 expression on neutrophils in patient 2 just before discharge.

Although significant comorbidities (such as those our patient 1 had) have been associated with poor outcomes in COVID-19, not all patients with such comorbidities will develop a severe disease. Our findings could help to identify those with a poorer prognosis in addition to those known risk factors. In fact, both soluble Gal-9 and TIM-3 expression on T cells was evaluated in samples from a small cohort of COVID-19 patients with disparate evolution and similar comorbidities (online suppl. Table 1). Our data indicate that the Gal-9/TIM-3 axis was upregulated in those patients who showed the worst evolution. It is important to remark that the samples were taken on admission and before any treatment.

In a new disease such as COVID-19, with neither previous clinical experience nor tools for patient classification and only two drugs, remdesivir and dexamethasone, indicated to be effective for selected patients [24,25], it is mandatory to explore and establish biomarkers to identify those patients at high risk. Here, we have presented data that show the potential of the Gal-9/TIM-3 axis for the
Martín-Quirós et al. 
classification of patients with a poor prognosis. Likewise, there is the possibility of its use as a pharmaceutical target in this clinical context. Furthermore, due to the functional implications of Gal-9/TIM-3 axis-blocking antibodies, these molecules should be explored for potential therapies for COVID-19. Notwithstanding, it is worth noting that the small cohort observed could constitute a limitation to the scope of our results and that the mechanisms underlying the described associations remain largely unknown.

Our study points to immune biomarkers that could play a key role in managing COVID-19 in this clinical setting, either for early recognition of patients with the potential for severe illness before clinical impairment presents itself or for adapting and individualizing treatments according to the progression of these biomarkers.

\section{Statement of Ethics}

The study was approved by the Human Ethics Committee of the University Hospital La Paz (PI-4100). Written informed consent was obtained from the patients for publication of this case report and any accompanying images. The healthy controls also signed an informed consent form. The data were treated according to the recommended confidentiality criteria and following the ethical guidelines of the 1975 Declaration of Helsinki.

\section{Conflict of Interest Statement}

All authors declare no conflicts of interest.

\section{Funding Sources}

The study was funded with grants to E. López-Collazo (COVID-19-ELC) by Fundación Alonso, Fundación Mutua Madrileña, Fundación Banco Santander, Fundación Uría, and City Hall of Madrid. K. Montalbán-Hernández was supported by the European Union's Horizon 2020 research and innovation program under the Marie Skłodowska-Curie grant agreement (No. 713673; "LaCaixa" Foundation).

\section{Author Contributions}

E.L.-C. designed the study; E.L.-C., A.M.-Q., and L.A.A. wrote the manuscript; A.M.-Q., C.M.-E., M.A.G.-G., Á.d.B.-C., and E.M.d.V. recruited the patients and followed them up; J.A.-O., V.T., K.M.-H., and R.L.-R. performed the cytometry analysis and the immunological biomarker quantification; C.R. performed the clustering analysis of TIM- 3 expression on $\mathrm{CD} 4^{+}$and $\mathrm{CD}^{+}$populations; E.L.-C., J.A.-O., and R.L.-R. discussed the results; J.V.Q., C.C.-Z., and K.M.-H. performed a critical review of the manuscript. All authors read and agreed to submit the manuscript for publication.

\section{References}

1 Qin C, Zhou L, Hu Z, Zhang S, Yang S, Tao Y, et al. Dysregulation of Immune Response in Patients with Coronavirus 2019 (COVID-19) in Wuhan, China. Clin Infect Dis. 2020 Jul; 71(15):762-8.

2 Xiong Y, Liu Y, Cao L, Wang D, Guo M, Jiang A, et al. Transcriptomic characteristics of bronchoalveolar lavage fluid and peripheral blood mononuclear cells in COVID-19 patients. Emerg Microbes Infect. 2020 Dec;9(1): 761-70.

3 Liu J, Li S, Liu J, Liang B, Wang X, Wang H, et al. Longitudinal characteristics of lymphocyte responses and cytokine profiles in the peripheral blood of SARS-CoV-2 infected patients. EBioMedicine. 2020 May;55:102763.

4 Zhou F, Yu T, Du R, Fan G, Liu Y, Liu Z, et al. Clinical course and risk factors for mortality of adult inpatients with COVID-19 in $\mathrm{Wu}$ han, China: a retrospective cohort study. Lancet. 2020 Mar;395(10229):1054-62.

5 López-Collazo E, Avendaño-Ortiz J, MartínQuirós A, Aguirre LA. Immune Response and COVID-19: A mirror image of Sepsis. Int J Biol Sci. 2020 Jul;16(14):2479-89.

6 Zheng M, Gao Y, Wang G, Song G, Liu S, Sun $\mathrm{D}$, et al. Functional exhaustion of antiviral lymphocytes in COVID-19 patients. Cell Mol Immunol. 2020 May;17(5):533-5.
7 Liu Y, Pang Y, Hu Z, Wu M, Wang C, Feng Z, et al. Thymosin Alpha 1 Reduces the Mortality of Severe Coronavirus Disease 2019 by Restoration of Lymphocytopenia and Reversion of Exhausted T Cells. Clin Infect Dis. 2020 Nov; 71(16):2150-7.

8 Zhou Y, Fu B, Zheng X, Wang D, Zhao C, Qi $\mathrm{Y}$, et al. Pathogenic T-cells and inflammatory monocytes incite inflammatory storms in severe COVID-19 patients. Natl Sci Rev. 2020 Mar;7(6):998-1002.

9 Kong Y, Wang Y, Wu X, Han J, Li G, Hua M, et al. Storm of soluble immune checkpoints associated with disease severity of COVID19. Signal Transduct Target Ther. 2020 Sep; 5(1):192.

10 Thevarajan I, Nguyen THO, Koutsakos M, Druce J, Caly L, van de Sandt CE, et al. Breadth of concomitant immune responses prior to patient recovery: a case report of non-severe COVID-19. Nat Med. 2020 Apr; 26(4):453-5.

11 Diao B, Wang C, Tan Y, Chen X, Liu Y, Ning $\mathrm{L}$, et al. Reduction and Functional Exhaustion of T Cells in Patients with Coronavirus Disease 2019 (COVID-19). Front Immunol. 2020 May; 11:827.

12 Kermali M, Khalsa RK, Pillai K, Ismail Z, Harky A. The role of biomarkers in diagnosis of COVID-19 - a systematic review. Life Sci. 2020 Aug; $254: 117788$.

13 Weiskopf D, Schmitz KS, Raadsen MP, Grifoni A, Okba NMA, Endeman H, et al. Phenotype and kinetics of SARS-CoV-2-specific T cells in COVID-19 patients with acute respiratory distress syndrome. Sci Immunol. 2020 Jun;5(48):eabd2071.

14 Guan WJ, Liang WH, Zhao Y, Liang HR, Chen ZS, Li YM, et al. Comorbidity and its impact on 1590 patients with COVID-19 in China: a nationwide analysis. Eur Respir J. 2020 May;55(5):2000547.

15 Nakeshbandi M, Maini R, Daniel P, Rosengarten S, Parmar P, Wilson C, et al. The impact of obesity on COVID-19 complications: a retrospective cohort study. Int $J$ Obes (Lond). 2020 Sep;44(9):1832-7.

16 Takahashi T, Ellingson MK, Wong P, Israelow B, Lucas C, Klein J, et al.; Yale IMPACT Research Team. Sex differences in immune responses that underlie COVID-19 disease outcomes. Nature. 2020 Dec;588(7837): 315-20.

17 Nishiga M, Wang DW, Han Y, Lewis DB, Wu JC. COVID-19 and cardiovascular disease: from basic mechanisms to clinical perspectives. Nat Rev Cardiol. 2020 Sep;17(9):54358. 
18 Kai H, Kai M. Interactions of coronaviruses with ACE2, angiotensin II, and RAS inhibitors-lessons from available evidence and insights into COVID-19. Hypertens Res. 2020 Jul;43(7):648-54.

19 Miesbach W. Pathological Role of Angiotensin II in Severe COVID-19. TH Open. 2020 Jun;4(2):e138-44.

20 Sehrawat S, Suryawanshi A, Hirashima M, Rouse BT. Role of Tim-3/Galectin-9 Inhibitory Interaction in Viral-Induced Immunopathology: Shifting the Balance toward Regulators. J Immunol. 2009 Mar;182(5):3191201.
21 Nebbia G, Peppa D, Schurich A, Khanna P, Singh HD, Cheng Y, et al. Upregulation of the Tim-3/Galectin-9 Pathway of T Cell Exhaustion in Chronic Hepatitis B Virus Infection. PLoS One. 2012;7(10):e47648.

22 Wykes MN, Lewin SR. Immune checkpoint blockade in infectious diseases. Nat Rev Immunol. 2018 Feb;18(2):91-104.

23 Vega-Carrascal I, Bergin DA, McElvaney OJ, McCarthy C, Banville N, Pohl K, et al. Galectin-9 signaling through TIM-3 is involved in neutrophil-mediated Gram-negative bacterial killing: an effect abrogated within the cystic fibrosis lung. J Immunol. 2014 Mar;192(5): 2418-31.
24 Horby P, Lim WS, Emberson JR, Mafham M, Bell JL, Linsell L, et al.; RECOVERY Collaborative Group. Dexamethasone in Hospitalized Patients with Covid-19 - Preliminary Report. N Engl J Med. 2020, Online ahead of print.

25 Spinner CD, Gottlieb RL, Criner GJ, Arribas López JR, Cattelan AM, Soriano Viladomiu A, et al.; GS-US-540-5774 Investigators. Effect of Remdesivir vs Standard Care on Clinical Status at 11 Days in Patients With Moderate COVID-19: A Randomized Clinical Trial. JAMA. 2020 Sep;324(11):1048-57. 